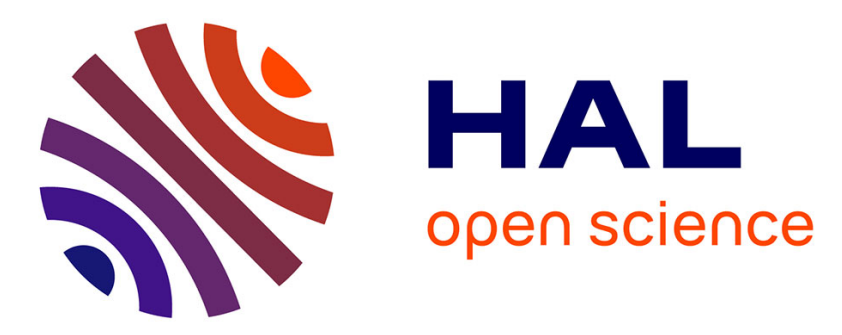

\title{
Efficient iterative receiver for Bit-Interleaved Coded Modulation according to the DVB-T2 standard
}

Meng Li, Charbel Abdel Nour, Christophe Jego, Jianxiao Yang, Catherine Douillard

\section{- To cite this version:}

Meng Li, Charbel Abdel Nour, Christophe Jego, Jianxiao Yang, Catherine Douillard. Efficient iterative receiver for Bit-Interleaved Coded Modulation according to the DVB-T2 standard. ICASSP 2011 IEEE 36th international Conference on Acoustics, Speech and Signal Processing, May 2011, Prague, Czech Republic. pp.3168 - 3171. hal-00617884

\section{HAL Id: hal-00617884 https://hal.science/hal-00617884}

Submitted on 30 Aug 2011

HAL is a multi-disciplinary open access archive for the deposit and dissemination of scientific research documents, whether they are published or not. The documents may come from teaching and research institutions in France or abroad, or from public or private research centers.
L'archive ouverte pluridisciplinaire HAL, est destinée au dépôt et à la diffusion de documents scientifiques de niveau recherche, publiés ou non, émanant des établissements d'enseignement et de recherche français ou étrangers, des laboratoires publics ou privés. 


\title{
EFFICIENT ITERATIVE RECEIVER FOR BIT-INTERLEAVED CODED MODULATION ACCORDING TO THE DVB-T2 STANDARD
}

\author{
Meng Li ${ }^{1}$, Charbel Abdel Nour ${ }^{1}$, Christophe Jégo ${ }^{2}$, Jianxiao Yang ${ }^{1}$ and Catherine Douillard ${ }^{1}$ \\ ${ }^{1}$ Institut Telecom, Telecom Bretagne, CNRS Lab-STICC UMR 3192 \\ Technopôle Brest Iroise, CS 8381829238 Brest \\ Université Européenne de Bretagne, France \\ firstname.lastname@telecom-bretagne.eu \\ ${ }^{2}$ IPB, ENSEIRB-MATMECA, CNRS IMS UMR 5218 \\ 351, Cours de la Libération, 33405 Talence \\ Université de Bordeaux, France \\ christophe.jego@ims-bordeaux.fr
}

\begin{abstract}
Bit-Interleaved Coded Modulation (BICM) offers a significant improvement in error correcting performance for coded modulations over fading channels compared to the previously existing techniques. Iterative processing at the receiver side can provide additional improvement to the BICM performance. In this paper, an efficient shuffled iterative receiver is investigated for the second generation of the terrestrial digital video broadcasting standard DVB-T2. The main contribution is scheduling an efficient message passing algorithm with low latency between the demapper and the LDPC decoder. A BER performance comparison between a fixed-point version that considers architectural constraints and a theoretical version over a fading channel with erasure is presented. It validates the potential of iterative receiver as practical and competitive solution for the DVB-T2 standard.
\end{abstract}

Index Terms - BICM, shuffled iterative receiver, $L D P C$ code, rotated constellation, DVB-T2 standard

\section{INTRODUCTION}

Signal Space Diversity (SSD) [1-2] doubles the diversity order of the conventional BICM schemes and largely improves the fading performance especially for high coding rate systems [3]. When a severe fading occurs, BICM with SSD [3] avoids the simultaneous fading in both the I and Q components leading to important gains. This scheme has been adopted by the second generation of the terrestrial digital video broadcasting standard (DVB-T2).

In order to achieve additional improvement in performance, iterations between the decoder output and the demapper (BICM-ID) can be introduced. BICM-ID with an outer LDPC code was investigated for different DVB-T2 transmission scenarios [3]. It is shown that an iterative processing associated with SSD can provide additional error correction capability reaching more than $1.0 \mathrm{~dB}$ over some types of channels. Thanks to these advantages, BICM-ID has been recommended in the DVB-T2 implementation guidelines [4] as a candidate solution to improve the performance at the receiver.

However, designing a low complexity high throughput iterative receiver remains a challenging task. One critical problem is the additional latency introduced by this additional iterative loop. Therefore, a more efficient information exchange method between the demapper and the decoder has to be applied. Another critical problem is the computation complexities of both the rotated QAM demapper and the LDPC decoder. In [5], a flexible demapper architecture for DVB-T2 is presented. Lowering complexity is achieved by decomposing the rotated constellation into two-dimensional sub-regions in signal space. In [6], a novel complexity-reduced LDPC decoder architecture based on the vertical layered schedule [7] and the normalized Min-Sum (MS) algorithm is detailed. It closely approaches the full-complexity BP performance provided in the implementation guidelines of the DVB-T2 standard. An additional critical problem is dealing with memory conflicts in the presence of double-diagonal, triplediagonal or multiple-diagonal sub-matrices within the parallel decoding units. They could introduce performance loss when the conflicts are not properly solved [8].

Since the vertical layered schedule enables parallel LDPC decoding, this schedule can be extended to the demapping process. Circuits continuously exchanging information between the Soft-Input Soft-Output (SISO) decoder on one hand and the demapper on the other hand are appealing to the implementation. In this context, processing one frame can be decomposed into multiple parallel smaller sub-frame processing having each a length equal to the parallelism level. While having a comparable computational complexity as the standard iterative schedule, the receiver with a shuffled iterative schedule enjoys a lower latency. However, such a parallel processing requires good matching between the demapping and the decoding processors in order to guarantee a high throughout pipeline architecture. This calls for an efficient message passing between both sides. In this paper, different schedule solutions are investigated for the DVB-T2 standard.

The remainder of the paper is organized as follows. In Section 2, the basic principles of the BICM-ID and SSD are briefly recalled. The detection principle for rotated constellation and a vertical layered decoding using a normalized MS algorithm are also introduced. In Section 3, hardware oriented iterative processing for the BICM receiver is detailed. Finally, simulation results validate the potential of the proposed iterative receiver for the DVB-T2 standard. 


\section{BICM-ID STRUCTURE}

\subsection{BICM-ID with SSD}

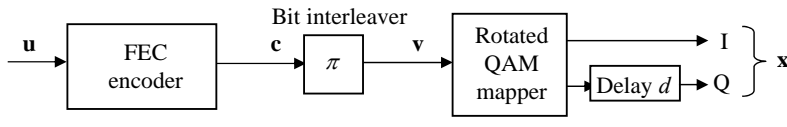

(a)

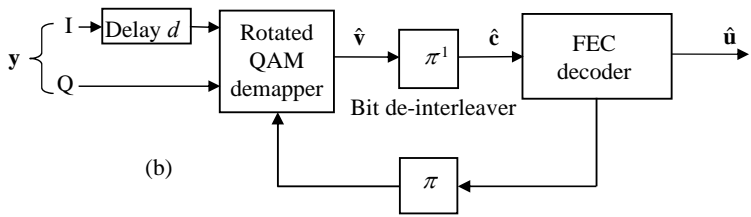

Fig. 1: (a) The BICM with SSD transmitter;

(b) Conventional BICM-ID receiver.

The SSD introduces two modifications to the classical BICM system, which are shown in Fig. 1. The classical QAM constellation is rotated by a fixed angle $\alpha$ and becomes a rotated constellation whose $\mathrm{Q}$ component is delayed for $d$ symbol periods, the delayed $\mathrm{Q}$ component and the current I component consist of a new complex symbol [3]. The in-phase and quadrature components of the classical QAM constellation are sent into two different rotated QAM symbols therefore doubling the constellation diversity of the BICM scheme. When a severe fading occurs, one of the components is erased and the according LLRs could be rescued from the remaining component. At the transmitter side, the messages $\mathbf{u}$ are encoded as the codeword c . Afterwards, this codeword $\mathbf{c}$ is interleaved by $\pi$ and becomes the input sequence $\mathbf{v}$ of the mapper. At each symbol time $t, m$ consecutive bits of the interleaved sequence $\mathbf{v}$ are mapped into complex symbol $x_{t}$. At the receiver side, the demapper calculates a two-dimensional squared Euclidean distance to obtain the bit LLR $\hat{v}_{t}^{i}$ of the $i^{\text {th }}$ bit of symbol $v_{t}$. These demapped LLRs are then deinterleaved and used as inputs of the decoder. The extrinsic information is finally generated by the decoder and fed back to the demapper for additional iterations.

\subsection{Demapping algorithm for iterative receiver}

For Gray-mapped QAM constellations, the demapper calculates two-dimensional Euclidean distance for the computation of the LLR $\hat{v}_{t}^{i}$ related to the $i^{\text {th }}$ bit of $v_{t}$. The resulting $\hat{v}_{t}^{i}$ becomes:

$\hat{v}_{t}^{i}=\sum_{x_{t} \in \chi_{0}^{i}} \oplus\left\{-\frac{D_{e u c}\left(x_{t}\right)}{\sigma_{w}^{2}}+\sum_{j=0, j \neq i, b_{j}=0}^{m-1} e x t_{j}^{(t)}\right\}-\sum_{x_{t} \in \chi_{1}^{j}} \oplus\left\{-\frac{D_{\text {euc }}\left(x_{t}\right)}{\sigma_{w}^{2}}+\sum_{j=0, j \neq i, b_{j}=0}^{m-1} e x t_{j}^{(t)}\right\}$

where $D_{\text {euc }}\left(x_{t}\right)$ is the square of the Euclidean distance between the constellation point and the equalized observation, i.e,

$$
D_{\text {euc }}\left(x_{t}\right)=\left[\rho_{t-d}\left(y_{e q, t-d}^{I}-x_{t-d}^{I}\right)\right]^{2}+\left[\rho_{t}\left(y_{e q, t}^{Q}-x_{t}^{Q}\right)\right]^{2}
$$

the operator $\oplus$ means the Jacobian logarithm, i.e.,

$$
x \oplus y=\left\{\begin{array}{c}
\max (x, y)+\log (1+\exp (-|x-y|)), \text { if }|x-y| \leq 5 \\
\max (x, y)+\log (1+\exp (-5)), \text { else }
\end{array}\right.
$$

ext $t_{j}^{(t)}$ is the a priori information of the $i^{\text {th }}$ mapping bit $b^{i}$ of the symbol $x_{t}$ and is also the decoded extrinsic information from (9). $y_{\text {eq,t-d }}^{I}$ and $y_{\text {eq,t }}^{Q}$ respectively represent the inphase and quadrature components of the equalized complex symbol $y_{e q, t} \cdot \rho_{t}$ is a scalar representing the channel attenuation at time $t$. $\chi_{b}^{i}$ represents the subset of constellation symbols with $i^{\text {th }}$ bit $b^{i}=b, b \in\{0,1\} \cdot \sigma^{2}$ is the AWGN variance.

\subsection{LDPC decoding algorithm for iterative receiver}

A vertical layered schedule (VSS) with the BP algorithm updates the messages between check and bit nodes in a column by column way as explained in [6].

$$
\begin{aligned}
& \text { Vertical layered BP algorithm } \\
& 0 \text {. Initialization: } \\
& \text { 1. } T_{m n}{ }^{(0)}=l l r_{n} \quad m \in M(n) \\
& \text { 2. } \quad \alpha_{m}^{(0)}=\prod_{m \in M(n)} \operatorname{sgn}\left(l l r_{n}\right), \beta_{m}^{(0)}=\sum_{m \in M(n)} \varphi\left(\left|l l r_{n}\right|\right)
\end{aligned}
$$

10. Hard decision according to $\operatorname{sgn}\left(T_{n}^{(t)}\right)$

where $l l r_{n}$ denotes the intrinsic channel reliability value of the bit node $n, E_{m n}$ denotes the message sent from check node $m$ to bit node $n, T_{m n}$ denotes the message sent from bit node $n$ to check node $m$ and $T_{n}$ denotes a posteriori information of bit node $n$.

\section{ITERATIVE RECEIVER FOR DVB-T2}

\subsection{Hardware oriented iterative algorithm}

To reduce the computation complexity of (1), a sub-region selection algorithm [5] is proposed to avoid a complete search of signals in the constellation plane. However, when iterative processing is considered, the sub-region selection algorithm becomes sub-optimal. In fact, the selected region by the algorithm may not contain the minimum Euclidean distance due to the extrinsic information. Therefore, the Maxlog approximation is the only complexity-reduced demapping approach applied in this case. 
On the LDPC side, a VSS Min-Sum (VSSMS) was proposed in [6]. It introduces only a $0.1 \sim 0.2 \mathrm{dBs}$ penalty with respect to VSS BP while greatly reducing complexity. However, in the context of BICM-ID, the VSSMS introduces an additional penalty and reduces the expected performance gain. In fact, a decoding algorithm with a higher accuracy is a must in this case. The Min-Sum-3 or VSSMS3 seems to provide the required precision at the lowest impact on complexity. The difference between the VSSMS and VSSMS3 is that the $3^{\text {rd }}$ minimum values are updated and saved leading to more accuracy of the check node process $M_{m}^{1}$.

The crucial problem in the implementation of a frame-byframe schedule in the iterative receiver is the latency introduced by the block interleaver and block de-interleaver. To overcome this problem, two new solutions are proposed. The first consists of replacing the classical RAM based block interleaver and de-interleaver memorizing the connections between the demapper output and the decoder input by a Look-Up-Table (LUT). The other is the application of VSS decoding to replace of the classical layered HSS LDPC decoding. In this way, both the decoded and demapped extrinsic information could be exchanged without waiting for the complete frame processing.

\subsection{Shuffled demapping and decoding algorithm}

The shuffled demapping and decoding algorithm is detailed as follows. Fig.2 also gives a graphical illustration.

\section{Shuffled Parallel Demapping and Decoding Algorithm}

For iteration $\forall t=1,2, \ldots, t_{\max }$, within one parallel processing unit with

$Q$ bits ( $Q$ can be 1, 45, 90,120, 180 or 360), performing the following algorithm for the current code bit index $n=1,2, \ldots, Q$ that corresponds to interleaved bit index $i=\pi(1), \pi(2), \ldots, \pi(Q)$, where $i=\pi(n)$ :

Demapper processing for $\forall i=\pi(1), \pi(2), \ldots, \pi(Q)$

(observation node processing)

$$
\begin{aligned}
\hat{v}_{t}^{i} \approx \max _{x_{t} \in \chi_{0}^{i}}\left\{-\frac{1}{\sigma_{w}^{2}} D_{\text {euc }}\left(x_{t}\right)+\sum_{j=0, j \neq i, b_{j}=0}^{m-1} e x t_{j}^{(t)}\right\}- \\
\max _{x_{t} \in \chi_{1}^{i}}\left\{-\frac{1}{\sigma_{w}^{2}} D_{\text {euc }}\left(x_{t}\right)+\sum_{j=0, j \neq i, b_{j}=0}^{m-1} e x t_{j}^{(t)}\right\}
\end{aligned}
$$

Decoder processing for $\forall n=1,2, \ldots, Q$

(check node processing)

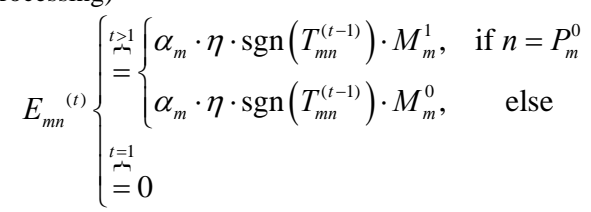

(bit node processing)

$$
\begin{gathered}
L L R_{n}=\hat{v}_{t}^{i}, \quad \text { where } n=\pi^{-1}(i) \\
T_{n}^{(t)}=\left\{\begin{array}{cc}
L L R_{n}, & t=1 \\
L L R_{n}+\sum_{m \in M(n)} E_{m n}{ }^{(t)}, & \text { else } \\
T_{m n}^{(t)}=T_{n}^{(t)}-E_{m n}^{(t)} &
\end{array}\right.
\end{gathered}
$$

(bit node updating for next demapping)

$$
\operatorname{ext}_{n}^{(t)}=T_{n}^{(t)}-L L R_{n}
$$

(check node updating)

$$
\begin{gathered}
\alpha_{m}=\alpha_{m} \cdot \operatorname{sgn}\left(T_{m n}^{(t-1)}\right) \cdot \operatorname{sgn}\left(T_{m n}^{(t)}\right), \quad m \in M(n) \\
\left\{\begin{array}{l}
M_{m}^{0}=\min _{1 \mathrm{st}}\left(\left|T_{m n}^{(t)}\right|,\left|T_{m k^{\prime}}^{(t-1)}\right|\right), \quad P_{m}^{0}=\operatorname{index}\left(M_{m}^{0}\right) \\
M_{m}^{1}=\min _{2 \mathrm{nd}}\left(\left|T_{m n}^{(t)}\right|,\left|T_{m k^{\prime}}^{(t-1)}\right|\right), \quad P_{m}^{1}=\operatorname{index}\left(M_{m}^{1}\right) \\
M_{m}^{2}=\min _{3 \mathrm{rd}}\left(\left|T_{m n}^{(t)}\right|,\left|T_{m k^{\prime}}^{(t-1)}\right|\right), \quad P_{m}^{2}=\operatorname{index}\left(M_{m}^{2}\right)
\end{array}\right.
\end{gathered}
$$

where $k^{\prime} \in N(m) \backslash n$.

The demappers perform (4) for the corresponding bits. Still for these updated bits, the decoding processors perform equation (5)-(11). Then another group of $Q$ bits are considered. The advantage of such a scheduling is a lower decoding latency leads to a decrease in the number of required iterations and/or better BER performance.

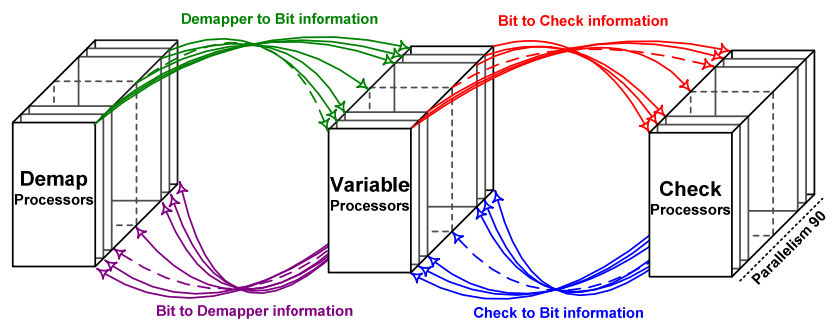

Fig.2. The basic idea of the parallelized iterative receiver

There are several possible message passing schedules. They correspond to the possible combinations of the parallelism of LDPC decoder and the message passing ways between the LDPC decoder and the demapper.

Table.1 different message passing schedules for the iterative receiver
\begin{tabular}{|c|c|c|c|}
\hline Schedule & A & B & C \\
\hline Receiver based on & Demapper & LDPC & LDPC \\
\hline Demapper symbols & 1 & $\leq 90$ & $\leq 90$ \\
\hline Updated LLR & $\log _{2}(M)$ & $\leq 90 \cdot \log _{2}(M)$ & 90 \\
\hline LDPC Parallelism & 1 & 90 & 90 \\
\hline LDPC bits processed & $\log _{2}(M)$ & 90 & 90 \\
\hline Feedback Extrinsic & $\log _{2}(M)$ & 90 & 90 \\
\hline
\end{tabular}

There interesting cases are listed in Table.1 under consideration of implementation. Schedule A is based on the demapper, LDPC works in the VSS schedule serially. Each symbol leads to $\log _{2}(M)$ variable bits updating, then all the extrinsic information is fed back to the original symbol. Schedules B and C are based on VSS LDPC decoder, with parallelism of 90 . So 90 variable bits get updated and generate 90 extrinsic informations that are fed back to a maximum of 90 demapper symbols. If all bits originate from different symbols, then there are 90 demappers working in parallel benefiting from the extrinsic information to update LLRs. The difference between 
Schedule B and Schedule C is the number of the LLRs that are updated during the iterative processing at the demapper. Schedule C is the desired schedule for HW implementation.

\section{EXPERIMENTAL RESULTS}

The simulation is carried out for all the schedules. Two comparisons of simulated performance of BER for different decoding schemes (QPSK and 16QAM, a code rate $\mathrm{R}=4 / 5$ and 16,200 bit frames), are presented in Fig.3 and Fig. 4, with a maximum of 50 iterations. The channel model used to simulate and emulate the effect of erasure events is a modified version of the classical Rayleigh fading channel. More information about this model is given in [5].

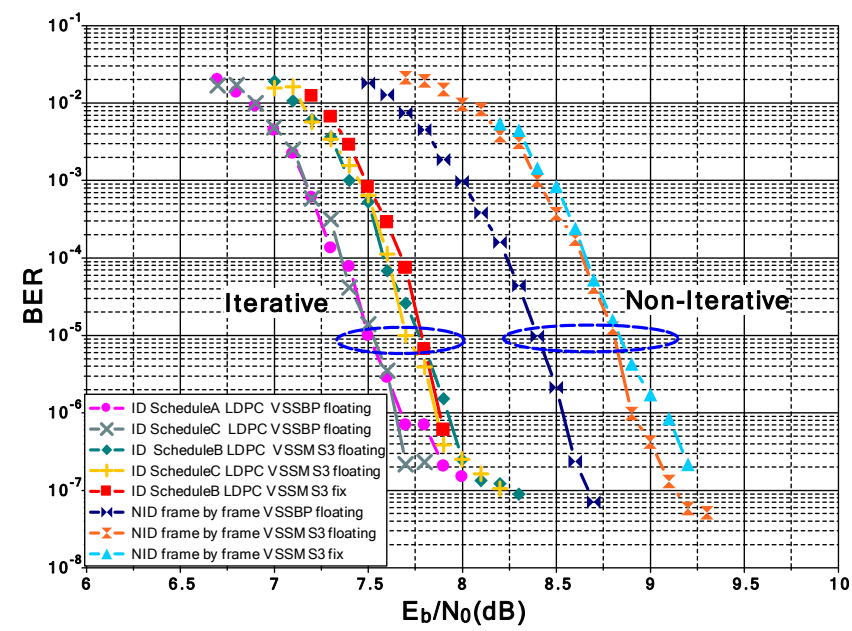

Fig. 3: Performance comparison for QPSK over a fading channel with $15 \%$ of erasures. $16 \mathrm{~K}$ frames, DVB-T2 LDPC, rate $\mathrm{R}=4 / 5$

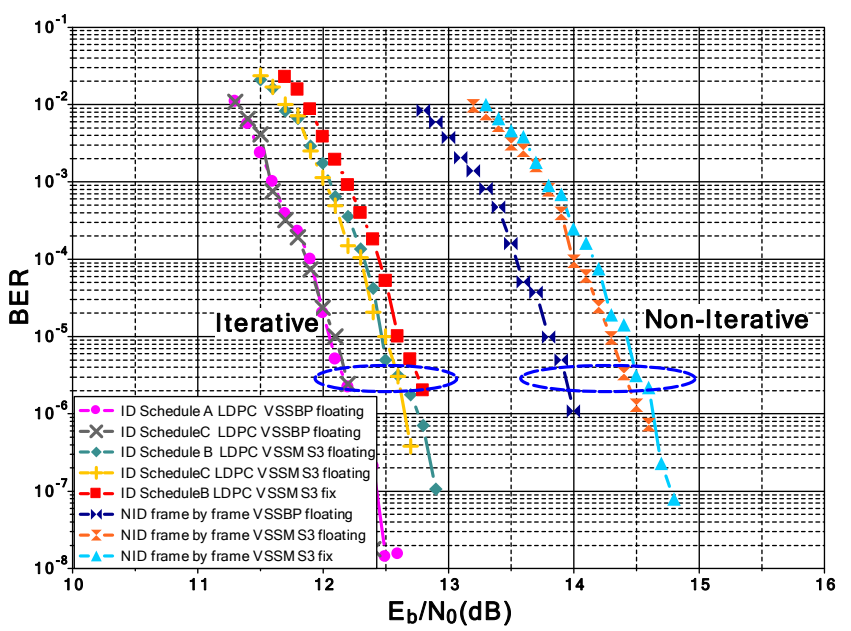

Fig. 4: Performance comparison for 16QAM over a fading channel with $15 \%$ of erasures. $16 \mathrm{~K}$ frames, DVB-T2 LDPC, rate $\mathrm{R}=4 / 5$

There is around 1.0 dB performance improvement @ 10e-6 of BER for the iterative floating point VSSBP receiver when compared to the non-iterative receiver. The gain increases to $2.0 \mathrm{~dB}$ when a 16QAM constellation is used. In a BICM-ID context, VSSMS3 enjoys a reduced penalty with respect to VSSBP. In other words, iterative processing between the decoder and the demapper seems to reduce the penalty of suboptimal LDPC decoding. In both cases, fixed point algorithms suffer from a small performance loss compared with floating point algorithms. However, this penalty is once again smaller for the iterative receiver.

\section{CONCLUSION}

In this paper, we have investigated possible scheduling of the BICM iterative receiver. It defines the order of passing messages between the demapper and the decoder. Our objective is to ensure a good matching between reception algorithms on one hand and the iterative receiver architecture on the other hand. Hardware-oriented simulated BER performance was given for two reception schemes over a fading channel with erasure. These results validate the potential of an iterative receiver as a practical and a competitive solution for the DVB-T2 standard. Currently, an FPGA prototyping to measure the performance of the proposed iterative receiver is under integration.

\section{ACKNOWLEDGMENT}

This work has been carried out in the framework of the SME42 project of the EUREKA's Eurostars programme.

\section{REFERENCES}

[1] X. Giraud, E. Boutillon, and J. C. Belfiore, "Algebraic tools to build modulation schemes for fading channels,” IEEE Trans. Commun., vol. 43, no. 3, pp. 938-952, May 1997.

[2] J. Boutros and E. Viterbo, "Signal space diversity: A powerand bandwidth-efficient diversity technique for the Rayleigh fading channel," IEEE Trans. Inform. Theory, vol. 44, no. 4, pp. 1453-1467, July 1998.

[3] C. Abdel Nour and C. Douillard, "Improving BICM performance of QAM constellations for broadcasting applications," $5^{\text {th }}$ Int. Symp. on Turbo Codes and Related Topics, Lausanne, Switzerland, pp. 55-60, Sep. 2008.

[4] DVB-T2, "Implementation guidelines for a second generation digital terrestrial television broadcasting system (DVB-T2)", draft ETSI TR 1028313 V0.10.00, Nov., 2009.

[5] M. Li, C. Abdel Nour, C. Jégo and C. Douillard, "Design of Rotated QAM Mapper/Demapper for the DVB-T2 Standard," SiPS 2009: IEEE workshop on Signal Processing Systems, October 2009.

[6] M. Li, C. Abdel Nour, C. Jégo and C. Douillard, "Design and FPFA prototyping of a bit-iterleaved coded modulation receiver for the DVB-T2 standard," SiPS 2010: IEEE workshop on Signal Processing Systems, October 2010.

[7] D. Hocevar, “ $A$ reduced complexity decoder architecture via layered decoding of LDPC codes," In Proc. Signal Processing Systems SIPS 2004, pp 107-112, October 2004.

[8] C. Marchand, J.-B. Dore, L. Conde-Canencia, E. Boutillon, "Conflict Resolution by Matrix Reordering for DVB-T2 LDPC Decoders", Globecom 2009, Haiwai, Dec. 2009. 